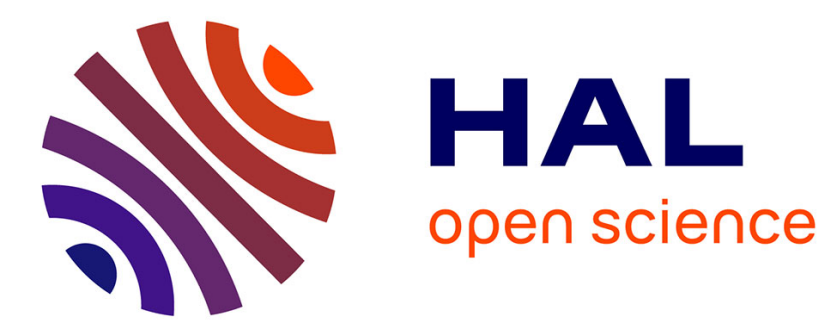

\title{
Numerical analysis of junctions between plates
}

Michel Bernadou, S. Fayolle, F. Lene

\section{To cite this version:}

Michel Bernadou, S. Fayolle, F. Lene. Numerical analysis of junctions between plates. RR-0865, INRIA. 1988. inria-00075689

\section{HAL Id: inria-00075689 https://hal.inria.fr/inria-00075689}

Submitted on 24 May 2006

HAL is a multi-disciplinary open access archive for the deposit and dissemination of scientific research documents, whether they are published or not. The documents may come from teaching and research institutions in France or abroad, or from public or private research centers.
L'archive ouverte pluridisciplinaire HAL, est destinée au dépôt et à la diffusion de documents scientifiques de niveau recherche, publiés ou non, émanant des établissements d'enseignement et de recherche français ou étrangers, des laboratoires publics ou privés. 


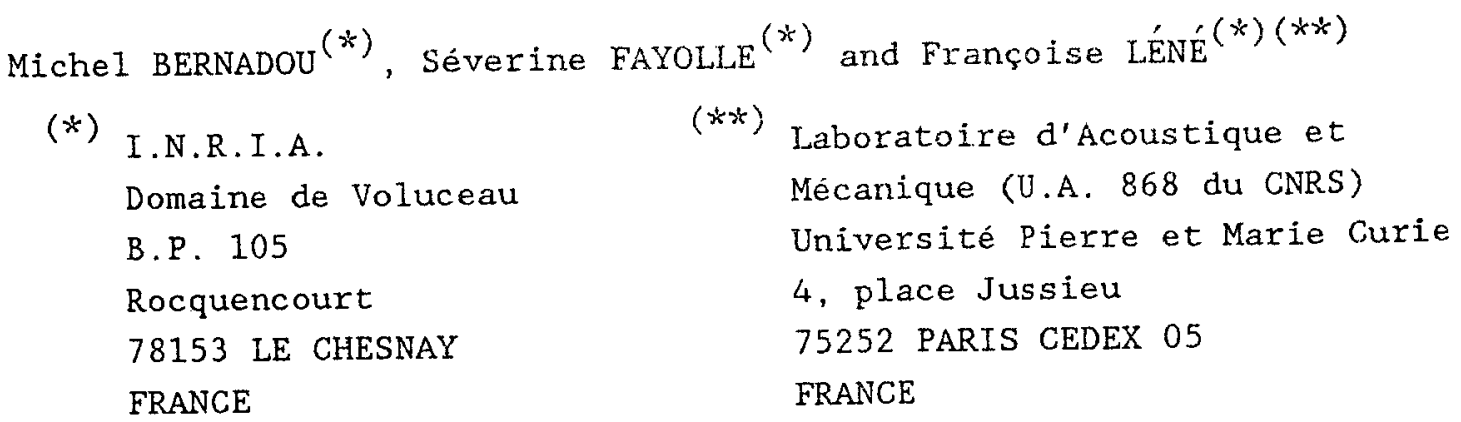

\section{SUMMARY}

The junctions of bars, plates and shells are the basic ingredients of any industrial structural construction. The numerical simulation of such junctions is a classical part of the commercial finite element codes.

On the other hand it seems that there are very few mathematical studies of such junctions. In this paper, we propose a variational formulation of plate junctions when these junctions can be considered as elastic or rigid hinges. Then, we study the mathematical properties of these equations as their approximation by finite element methods. We conclude by reporting some numerical experiments.

\section{ANALYSE NUMERIQUE DE JONCTIONS DE PLAQUES}

\section{RESUME}

Les jonctions de barres, plaques et coques constituent des éléments essentiels de toute construction industrielle de structures. La simulation numérique de telles jonctions est une composante classique de tout code commercial d'éléments finis.

Par contre, il semble que l'on dispose de très peu d'études mathématiques de telles jonctions. Dans ce travail, nous proposons une formulation variationnelle de jonctions de plaques lorsque ces jonctions peuvent être considérées comme des charnières élastiques ou rigides. On étudie ensuite les propriétés mathématiques de ces équations ainsi que leur approximation par des méthodes d'éléments finis puis on conclue en donnant quelques résultats numériques. 


\section{CONTENTS}

1 - INTRODUCTION

2 - MECHANICAL MODELLING

2.1 - Hypotheses and notations

2.2 - Junction of two plates

2.3 - The equations of the junction problem

3 - VARIATIONAL FORMULATIONS AND EXISTENCE RESULTS

3.1 - The case of an elastic hinge

3.2 - The case of a rigid hinge

3.3 - Study of the behaviour of the elastic hinge when $k \rightarrow \infty$

4 - APPROXIMATION BY CONFORMING FINITE ELEMENT METHODS

4.1 - Approximation in case of an elastic hinge

4.2 - Approximation in case of a rigid hinge

4.3 - Inplementation in case of elastic hinge

4.4 - Implementation in case of a rigid hinge

5 - NUMERICAL EXAMPLES

5.1 - Example of a folded cantilever plate with rigid hinge subjected to a line load at its tip

5.2 - Multifolded plate with rigid hinges

5.3 - Example of a folded cantilever plate with elastic hinge subjected to a line load at its tip

CONCLUSION

REFERENCES 


\section{1 - INTRODUCTION}

Many industrial constructions use, as basic components, elastic solids, bars, plates and shells. The numerical simulation of such assemblages needs a good approximation of each constitutive element as well as a good representation of their junctions. In engineering literature, there are many contributions on the best way to modelize and, particularly, to compute such constructions. Thus, for the modelization of the mechanical engineering aspects, we refer the reader to the chapter 6 of [1] which is devoted to multishell structures, while for finite element methods we can recommand [2] [3] and the references of these works.

By constrast there are very few mathematical studies in these directions. Let us mention some recent works [4] [5] [6] which are mainly concerned with the problem of the junction between three-dimensional and two-dimensional linearly elastic structures while [7] [8] consider asymptotic developments for junction of two plates.

In this paper we restrict our attention to the numerical analysis of the junction between two plates. Our study lies on the following assumptions :

- small deformations

- elastic, homogeneous, isotropic matesial

- deformation through the thickness obeyes the KIRCHHOFF-LOVE hypothesis

- the junction can be assimilated to a rigid or an elastic hinge.

The contents of this paper can be outline as follows. Section 2 discusses the mechanical modelling of the junction of plates in terms of partial differential equations. We start by recalling the general KIRCHHOFF-LOVE plate equations and then we introduce the modelization of the junction as an elastic or a rigid hinge. Section 3 gives the variational formulations of these different junctions and the corresponding existence results. It is also proved that the elastic hinge becomes rigid when its stiffness becomes infinite. Section 4 contains the approximation by an approximate finite element method and Section 5 presents some numerical examples.

ACKNOWLEDGEMENTS : The authors are indebted to $\mathrm{Pr}$. G. DUVAUT for suggesting this approach and for several useful discussions. They also acknowledge ASULAB S.A. for partly supporting this study. 


\section{2 - MECHANICAL RODELLING}

In this paragraph, we introduce the iain notations and the basic equations that we will use subsequently.

\subsection{Hypotheses and notations}

This first section is devoted to the recalls concerning the equations of only one thin plate. Let $\mathbb{C}^{3}$ be the Euclidean space referred to the orthonormal system $\left(0, \vec{e}_{1}, \vec{e}_{2}, \vec{e}_{3}\right)$. Consider a plate whose middle surface lies in the plane $\left(0, \vec{e}_{1}, \vec{e}_{2}\right)$ and coincides with the domain $S \in \mathbb{R}^{2}$. We assume that the boundary $\partial S$ of $S$ is sufficiently smooth.

Classically, the displacement field of the current point $P\left(x_{1}, x_{2}, x_{3}\right)$ of the plate can be described by

(2.1.1) $\quad \overrightarrow{\mathrm{U}}\left(\mathrm{x}_{1}, \mathrm{x}_{2}, \mathrm{x}_{3}\right)=\left[\mathrm{u}_{\alpha}\left(\mathrm{x}_{1}, \mathrm{x}_{2}\right)+\mathrm{x}_{3} \dot{\ell}\left(\mathrm{x}_{1}, \mathrm{x}_{2}\right)\right] \overrightarrow{\mathrm{e}}_{\alpha}+\mathrm{u}_{3}\left(\mathrm{x}_{1}, \mathrm{x}_{2}\right) \overrightarrow{\mathrm{e}}_{3}$

where

$$
\text { (2.1.2) } \quad \vec{\ell}=\vec{R} \times \vec{e}_{3} \text {, }
$$

$\vec{\Omega}$ denoting the infinitesimal rotation vector.

In relation (2.1.1) and suosequentiy, we use the summation convention on repeated indices. Latin indices $i, j, \ldots$ take their values in the set $(1,2,3)$ while greek indices $\alpha, \beta, \ldots$ take their values in the set $\{1,2\}$.

Then, the strain tensor is given by $i$ ts components upon the basis $\left(\vec{e}_{1}, \vec{e}_{2}, \vec{e}_{3}\right)$ :

(2.1.3) $D_{\alpha \beta}=e_{\alpha \beta}+x_{3} K_{\alpha \beta} ; 2 D_{\alpha 3}=l_{\alpha}+u_{3, \alpha} ; D_{33}=0$

where

$$
2 e_{\alpha \beta}(\vec{u})=u_{\alpha, \beta}+u_{\beta, \alpha} ; 2 \mathrm{~K}_{\alpha \beta}=\ell_{\alpha, \beta}+\ell_{\beta, \alpha}
$$

By using the KIRCHHOFF-LOVE assumption $\left(D_{\alpha_{3}}=0\right)$, we obtain that the non zero components of the strain field are determined by (2.1.3) from the knowledge of both symmetric tensor fields $e_{\alpha \beta}$ and $\mathrm{K}_{\alpha \beta}$. They can be expressed as functions of the components of the middle surface displacement as follows :

$$
2 e_{\alpha \beta}(\vec{u})=u_{\alpha, \beta}+u_{\beta, \alpha} ; k_{\alpha \beta}(\vec{u})=u_{3, \alpha \beta} .
$$

According to [9], the internal loads associated to the considered kinematic, are described by :

i) a symmetrical tensor of stress resultants $\mathrm{N}_{\alpha \beta}$;

ii) a symmetrical tensor of stress couples $M_{\alpha \beta}$. 
We assume that the external loads induce

i) a distribution of forces whose resultant is $\vec{p}=p_{i} \vec{e}_{i}$ on the middle surface $S$ and whose resultant moment is $\overrightarrow{0}$ on $S$;

ii) a distribution of forces whose resultant is $\vec{T}=T_{i} \vec{e}_{i}$ on $\partial S$ and whose resultant moment is $=l_{t}=C \|_{n} \vec{n}+c l_{t} \vec{t}$, where $\vec{n}$ denotes the unit external normal to $\partial S$ in the plane of $S$ and where $\vec{t}=\vec{e}_{3} \times \vec{n}$.

The equilibrium equations, obtained by the principle of virtual work, can be written as follows :

(2.1.6) $\quad \mathrm{N}_{\alpha \beta, \beta}+\mathrm{P}_{\alpha}=0$ in $\mathrm{s}$

(2.1.7) $\quad M_{\alpha \beta, \alpha \beta}+P_{3}=0$ in $S$

with the boundary conditions

$\left.\begin{array}{ll}(2.1 .8) & T_{3}=l_{n, t}=M_{\alpha \beta, \beta} n_{\alpha}+\left(M_{\alpha \beta} n_{\beta} t_{\alpha}\right), t \\ (2.1 .9) & T_{\alpha}=N_{\alpha \beta} n_{\beta} . \\ (2.1 .10) & =1 t_{t}=M_{\alpha \beta} n_{\alpha} n_{\beta}\end{array}\right\}$ on $\partial S$

where $n_{\alpha}$ and $t_{\alpha}$ are the components of vectors $\vec{n}$ and $\vec{t}$, i.e., $\vec{n}=n_{\alpha} \vec{e}_{\alpha}, \vec{\tau}=t_{\alpha} \vec{e}_{\alpha}$.

Finally, the deformations are. assumed to be small and governed by the equations of the linear elasticity, and the material is supposed to be homogeneous and isotropic. Then, the behaviour 1 gw of the plate is described by the relations isee [9]) :

(2.1.11) $\quad N_{\alpha \beta}=\frac{E e}{1-\nu^{2}}\left((1-\nu) e_{\alpha \beta}+\nu e_{\gamma \gamma}{ }_{\alpha \beta}\right)$

$(2.1 .12)$

$$
\left.\mathrm{M}_{\alpha \beta}=\frac{\mathrm{Ee}^{3}}{12\left(1-\nu^{2}\right)}(1-\nu) \mathrm{K}_{\alpha \beta}+\nu \mathrm{K}_{\gamma \gamma} \delta_{\alpha \beta}\right)
$$

where $e, E$ and $\nu$ denote respectively the thickness of the plate, the Younc modulus and the POISSON coefficient of the material.

\section{$\underline{2.2 \text { - Junction of two plates }}$}

Consider two plates with distinct middle surfaces $S$ and $S$ which joint along a common rectilinear boundary $\Gamma$ (see figure 2.2.1). From now on, we note $($.$) the$ quantities related to the plate $S$ while $(\dot{\sim})$ denotes the quantities related to the plate $\underline{\mathbf{S}}$.

Upon the boundaries $\partial S$ and $\partial \underline{S}$, we define two local direct orthonormal reference systems $\left(\vec{n}, \vec{t}, \vec{e}_{3}\right)$ and $\left(\overrightarrow{\underline{n}}, \overrightarrow{\underline{t}}, \vec{e}_{3}\right)$ which include as intrinsic vectors the unit external normals $\vec{n}$ and $\overrightarrow{\underline{n}}$ to the boundaries $\partial S$ and $\partial \underline{S}$ in the planes respectively determined by $S$ and $S$. If we define 
(2.2.1) $\quad \vec{t}=\vec{t}$ and $\overrightarrow{\underline{E}}_{3}=\overrightarrow{\mathbf{I}} \times \overrightarrow{\underline{t}}$.

then both lines $\partial S$ and $\partial \underset{\text { S }}{ }$ are positively oriented with respect to $\vec{e}_{3}$ and $\vec{e}_{3}$.

Finally, with respect to the reference system $\left(\vec{n}, \vec{t}, \vec{e}_{3}\right)$ attached to the common side $\Gamma$, we define the angle $\theta$ between both plates:

$(2.2 .2) \quad \theta-(\vec{n}, \vec{n}) \vec{t}$.

In particular, when both plates are coplanar and placed side by side, this angie is equal to $*$ while this angle is equal to 0 when the plates are superposed. All these geometrical considerations are illustrated on figure 2.2.1.

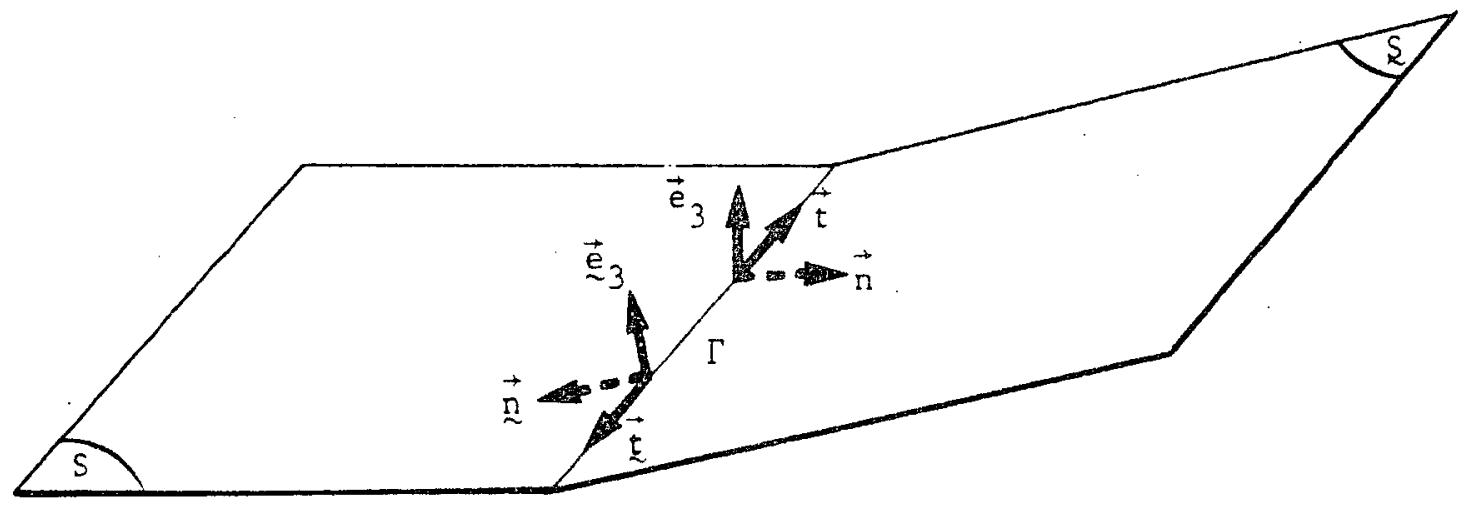

\section{Figure 2.2.1: Junction of two plates}

For any behaviour of the hinge $\Gamma$, the application of the action-reaction principle at any point of $\Gamma$, implies the transmission of the efforts, i.e..

$(2.2 .3) \quad \overrightarrow{\mathrm{I}}(\mathrm{M})=\overrightarrow{\mathrm{I}}(\mathrm{M})$ and $=\vec{l}(\mathrm{M})=\vec{l}(\mathrm{M}), \forall M \in \Gamma$.

In particular, note that $\overrightarrow{\|} \|(M)=\overrightarrow{\|}(M)$ on $r$ implies $=\left\|_{n}(M)==\right\|_{n}(M)=0$, $\forall M \in I$, so that: $\vec{l}(M)=\vec{l}(M)=-\|_{\tau}(M) \vec{t}, \forall M \in \Gamma$.

Subsequently, we examine two types of behaviours:

i) a rigid behaviour for which the continuity of the displacements and of the rotations is insured at any point $M$ of $\Gamma$, i.e. :

(2.2.4) $\left\{\begin{array}{l}\vec{u}(M)=\vec{u}(M) \\ \vec{\Omega}(M) \cdot \vec{t}-\vec{\Omega}(M) \cdot \vec{t}\end{array}\right.$

where $\vec{\Omega}$ (resp. $\vec{\Omega}$ ) is defined by relation (2.1.2);

ii) an elastic behaviour for which only the continuity of the displacements is insured at any point $M$ of $r$, i.e.,

$(2.2 .5)\left\{\begin{array}{l}\vec{u}(M)-\vec{u}(M) \\ =\|_{t}(M)--k[\vec{\Omega}(M)-\vec{\Omega}(M)] \cdot \vec{t} .\end{array}\right.$ 
Thus, the second equation of relation (2.2.4) is replaced by the requirement: the moment $\overrightarrow{C l}$ is proportional to the jump of the tangential components of rotations along the hinge $\Gamma$. The coefficient $k$ of elastic stiffness of the hinge is a given positive number attached to the problem into consideration.

\section{Junction conditions in terms of displacement components}

Let us consider the junction conditions (2.2.4) and (2.2.5) which are expressed in vectorial forms. By using the components of displacements and rotations upon the above reference systems, we are able to get the expression of the junction conditions in terms of components. At any point $M \in \Gamma$, let us set :

(2.2.6) $\left\{\begin{array}{l}\vec{u}(M)=u_{n} \vec{n}+u_{t} \vec{t}+u_{3} \vec{e}_{3} \\ \vec{u}(M)=\underline{u}_{\underline{n}} \overrightarrow{\underline{n}}+\underline{u}_{\underline{t}} \overrightarrow{\underline{t}}+\underline{\sim}_{3} \vec{e}_{3}\end{array}\right.$

Definitions (2.2.1) and (2.2.2) imply

$$
\vec{n}=\vec{n} \cos \theta-\vec{e}_{3} \sin \theta, \vec{t}=-\overrightarrow{\underline{t}}, \vec{e}_{3}=-\vec{n} \sin \theta-\vec{e}_{3} \cos \theta
$$

so that with $(2.2 .6)$ :

(2.2.7) ${\underset{\sim}{\underline{n}}}_{\underline{n}}=u_{n} \cos \theta-u_{3} \sin \theta, \underline{\underline{t}}_{\underline{t}}=-u_{t}, \underline{\sim}_{3}=-u_{n} \sin \theta-u_{3} \cos \theta$.

On the other hand, the assumption $D_{\alpha^{3}}=0$ involves $\ell_{\alpha}=-u_{3, \alpha}$ or, equivalently, $\hat{l}_{t}=-u_{3, t}$ and $\hat{l}_{n}=-u_{3, n}$. By setting

$$
\vec{n}=\Omega_{n} \vec{n}+\Omega_{t} \vec{t}+\Omega_{3} \vec{e}_{3} \text {, }
$$

the equation $\vec{l}=\vec{\Omega} \times \vec{e}_{3}=-\Omega_{n} \vec{t}+\Omega_{t} \vec{n}$ implies $\Omega_{n}=-l_{t}=u_{3, t}, \Omega_{t}=l_{n}=-u_{3, n}$ so that for any point $M \cdot \in \Gamma$ :

(2.2.8) $\quad \vec{n}(M)=u_{3, t} \vec{n}-u_{3, n} \vec{t}+\Omega_{3} \vec{e}_{3}$

Similarly, for any point $M \in \Gamma$ :

$$
\vec{\Omega}(M)=u_{3}, \underline{\underline{n}}-\underline{u}_{3, \underline{\sim}} \underset{\sim}{\vec{t}}+\Omega_{3} \overrightarrow{\mathrm{e}}_{3}
$$

so that the relation $\vec{t}=-\vec{t}$ implies

(2.2.9). $\vec{\Omega}(M)-\vec{\Omega}(M)=-\left(u_{3, n}+\underline{u}_{3, \underset{\sim}{n}}\right) \vec{t}+u_{3, t} \vec{n}-\underline{u}_{3, t} \underset{\sim}{\vec{n}}+\Omega_{3} \vec{e}_{3}-\Omega_{3} \vec{e}_{3}$

Thus, by assuming that we have a "real" junction $(\theta \neq k \pi, k \in \mathbb{Z})$, the vectors $(\vec{n}, \vec{\tau}, \vec{n})$ are independent. Then we have the following cases to consider:

i) Rigid hinge : From (2.2.9), condition $\vec{\Omega}(M) \cdot \vec{t}=\vec{\Omega}(M) \cdot \vec{t}$ implies on $\Gamma$ :

(2.2.10) $u_{3, n}+\underline{u}_{3, n}=0$;

ii) Elastic hinge : Relations (2.2.5) and (2.2.9) imply on $\Gamma$ :

(2.2.11) $k\left(u_{3, n}+\underline{u}_{3, \underline{n}}\right)=C U_{t}(M)$. 
Remark 2.2.1 : The rigid behaviour can be interpretated as the limit case $(k \rightarrow \infty)$ of the elastic behaviour of a hinge whose stiffness coefficient would be very large. We will come back to this result in section 3.3.

\section{3 - The equations of the junction problem}

In section 2.1, we have recalled the equations of a plate while, in section 2.2 : we have obtained the junction conditions of two plates both for rigid or elastic hinges, in vectorial forms and in terms of components.

For simplicity, we assume subsequently that the plate $S$ is clamped on a part $\partial S_{0}$ of its boundary, with measure $\left(\partial S_{0}\right)>0$, so that

$$
\vec{u}=\overrightarrow{0}, u_{3, n}=0 \text { on } \partial s_{0} \text {. }
$$

The complementary part of the boundary as is assumed to be such that

(2.3.1) $\quad \partial S-\partial S_{0}=\Gamma \cup \partial S_{1}$

where $r=\partial S \quad \partial S$ and where $\partial S_{1}$ is loaded by $(\vec{T}, c \overrightarrow{1})$. Likewise, we assume that

(2.3.2) $\left\{\begin{array}{l}\partial{\underline{S_{0}}}_{0}=\phi \quad(\text { not essential) } \\ \partial S_{=}=\Gamma \cup \partial \underline{S}_{1}\end{array}\right.$

where $\partial \underline{S}_{1}$ is loaded by $(\vec{I},=\vec{l}(\mathbb{C})$.

Then, by assembling all the previous results, the equations of this junction problem of plates are :

(2.3.3) $\quad \mathrm{N}_{\alpha \beta, \beta}+\mathrm{P}_{\alpha}=0, \mathrm{M}_{\alpha \beta, \alpha \beta}+\mathrm{p}_{3}=0$ in $\mathrm{S}$,

(2.3.4) $\quad \quad_{\alpha \beta, \beta}+\mathrm{R}_{\alpha}=0, \mathrm{M}_{\alpha \beta, \alpha \beta}+\mathrm{E}_{3}=0$ in $\underline{\mathrm{S}}$,

(2.3.5) $\vec{u}=\overrightarrow{0}, u_{3, n}=0 \quad$ on $\partial s_{\circ}$,

(2.3.6) $\quad T_{3}-\epsilon \mathcal{H}_{n, t}=M_{\alpha \beta, \beta} \mathrm{n}_{\alpha}+\left(M_{\alpha \beta} \mathrm{n}_{\beta} t_{\alpha}\right), t ; T_{\alpha}=N_{\alpha \beta} n_{\beta} ;$ $C \mathbb{H}_{t}=\mathrm{M}_{\alpha \beta} \mathrm{n}_{\alpha} \mathrm{n}_{\beta}$ on $\partial \mathrm{S}_{1}$

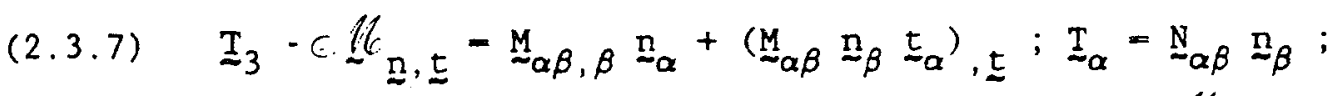
$C \underline{l l}_{\underline{t}}-\underline{M}_{\alpha \beta} \underline{n}_{\alpha} \underline{n}_{\beta}$ on $\partial \underline{S}_{1}$

and the conditions of junction upon $\Gamma$ which are depending on the type of hinge :

$\left.\begin{array}{ll}(2.3 .8) & \vec{u}-\vec{u} \text { on } \Gamma \\ (2.3 .9) & u_{3, n}+\underline{u}_{3, n}=0 \text { on } \Gamma\end{array}\right\}$ (rigid hinge) 
(2.3.10) $\quad \vec{u}=\vec{u}$ on $\Gamma$

(2.3.11) $k\left(u_{3, n}+u_{3, n}\right)=C t_{t}(M)$ on $r$

(elastic hinge)

\section{3 - VARIATIONAL FORMULATIONS AND ERISTENCE RESULTS}

From the equations stated in section 2.3, we derive the corresponding variational formulations in suitable spaces and then, we prove existence and uniqueness results. We conclude by examining the behaviour of the elastic hinge when $k \rightarrow+\infty$.

\section{1 - The case of an elastic hinge}

Let us consider the first plate $S$ independently of a possible junction with another plate S. By multiplying the equilibrium equations (2.1.6) and (2.1.7) by "test functions" $v_{\alpha}$ and $v_{3}$, by integrating by parts upon the middle surface $s$ and then, by taking into account the general boundary conditions (2.1.8) to (2.1.10), we obtain with (2.1.5)

$$
\left\{\begin{aligned}
& \int_{S}\left\{N_{\alpha \beta} e_{\alpha \beta}(\vec{v})+M_{\alpha \beta} K_{\alpha \beta}(\vec{v})\right\} d S \\
&=\int_{S} \vec{p} \cdot \vec{v} d S+\int_{\partial S}\left\{\vec{T} \cdot \vec{v}+C\left\|_{n} v_{3, t}-C\right\|_{t} v_{3, n}\right\} d s
\end{aligned}\right.
$$

Exactiy in the same way, we would get for the plate $\mathbf{S}$

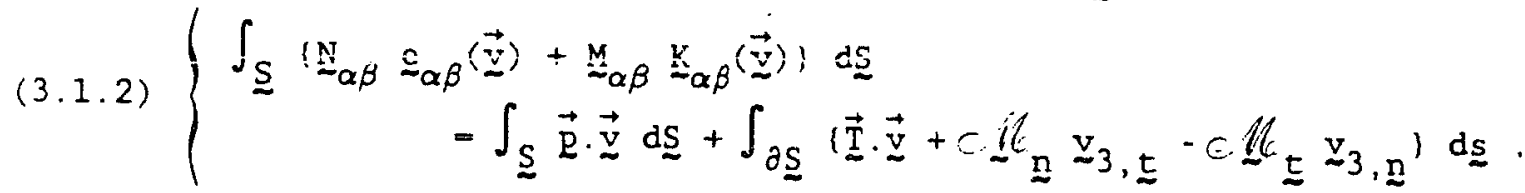

Now, we assume that both plates $S$ and $\underline{S}$ are such that:

i) the plate $S$ is clamped along $\partial S_{0}$, i.e., the conditions (2.3.5) are satisfied ;

ii) the plates $S$ and $\underline{S}$ are jointing along the common side $\Gamma$ so that conditions $[(2.3 .8),(2.3 .9)]$ or $[(2.3 .10),(2.3 .11)]$ are satisfied, depending of the type of hinge we are considering.

With notations (2.3.1) and (2.3.2), we obtain by adding equations (3.1.1) and $(3.1 .2)$ :

$(3.1 .3)$

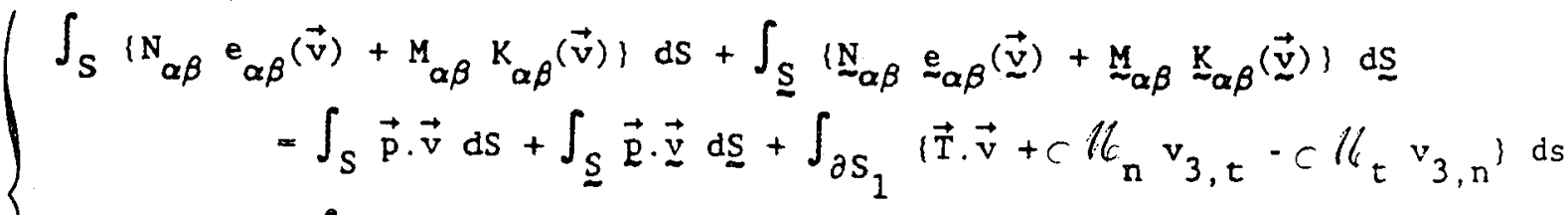

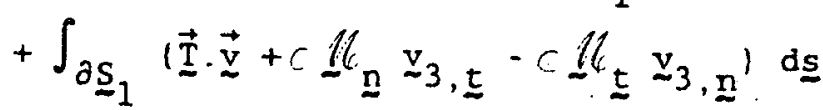

$$
\begin{aligned}
& +\int_{\Gamma}\left\{\overrightarrow{\mathrm{T}} \cdot(\overrightarrow{\mathrm{v}}-\overrightarrow{\mathrm{v}})-c \|_{t}\left(\mathrm{v}_{3, \mathrm{n}}+\mathrm{v}_{3, \mathfrak{n}}\right)\right\} \mathrm{d} \boldsymbol{\gamma} \text {. }
\end{aligned}
$$


To express the last integral we have used the properties (2.2.3) of transmission of the efforts, i.e., $\vec{T}=\vec{I}$ on $\Gamma$ and $C \vec{U}-C \mathscr{H}$ on $\Gamma$, that is equivalent to

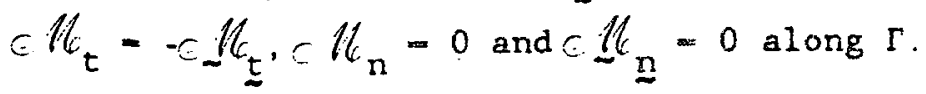

Let us emphasize that the expression (3.1.3) is valid for rigid hinge as well as for elastic hinge. Now let us specialize equation (3.1.3) to the case of an elastic hinge. In order to set up the variational formulation of the problem we have to:

i) substitute the behaviour laws (2.1.11) (2.1.12) (and similar relations for plate $\mathbf{S}$ ] into the equation (3.1.3);

ii) define a suitable admissible displacement space. In this way, we set:

(3.1.4) $\left\{\begin{array}{l}\mathrm{v}_{1}=\left(\mathrm{v} \in \mathrm{H}^{1}(\mathrm{~S}), \mathrm{v}=0 \text { on } \partial \mathrm{S}_{\mathrm{o}}\right) \\ \mathrm{v}_{2}=\left(\mathrm{v} \in \mathrm{H}^{2}(\mathrm{~S}), \mathrm{v}=\mathrm{v}_{\mathrm{n}}=0 \text { on } \partial \mathrm{S}_{\mathrm{o}}\right) \\ \overrightarrow{\mathrm{v}}=\mathrm{v}_{1} \times \mathrm{v}_{1} \times \mathrm{v}_{2}\end{array}\right.$

and

(3.1.5) $\quad \overrightarrow{\mathrm{V}}=\mathrm{H}^{1}(\underline{\mathrm{S}}) \times \mathrm{H}^{1}(\underline{\mathrm{S}}) \times \mathrm{H}^{2}(\underline{\mathrm{S}})$

so that the kinematically admissible displacement field for an elastic junction problem is

(3.1.6) $\quad \mathrm{W}_{E \ell}=((\vec{w} ; \vec{w}) \in \overrightarrow{\mathrm{V}} \times \overrightarrow{\mathrm{V}} ; \overrightarrow{\mathrm{w}}=\overrightarrow{\mathrm{w}}$ on $\Gamma)$

iii) substitute condition (2.3.11) into relation (3.1.3).

Then, the variational formulation of the problem can be stated as follows

$(3.1 .7)\left\{\begin{array}{l}\text { Find }(\vec{u} ; \vec{u}) \in W_{E \ell} \text { such that } \\ a[(\vec{u} ; \vec{u}),(\vec{v} ; \vec{v})]+k b[(\vec{u} ; \vec{u}),(\vec{v} ; \vec{v})]=\ell(\vec{v} ; \vec{v}) \\ \text { for any }(\vec{v} ; \vec{v}) \in W_{E \ell}\end{array}\right.$

where

$(3.1 .8)$

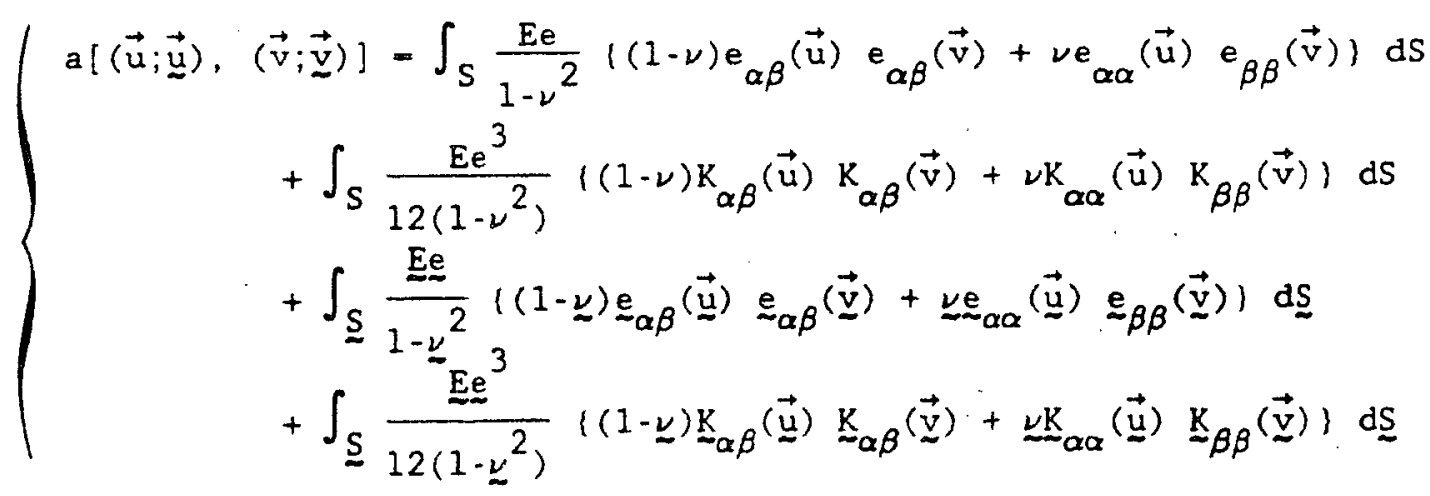

(3.1.9) b[( $\vec{u} ; \underline{\vec{u}}),(\vec{v} ; \overrightarrow{\underline{v}})]-\int_{\Gamma}\left(u_{3, n}+\underline{u}_{3, \underline{n}}\right)\left(v_{3, n}+\underline{v}_{3, \underline{n}}\right) d \gamma$ 


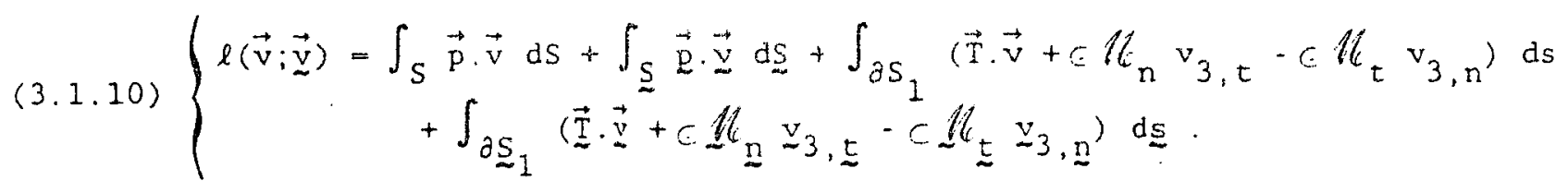

Thus, we obtain the following theorem:

Theorem 3.1.1: Assume that

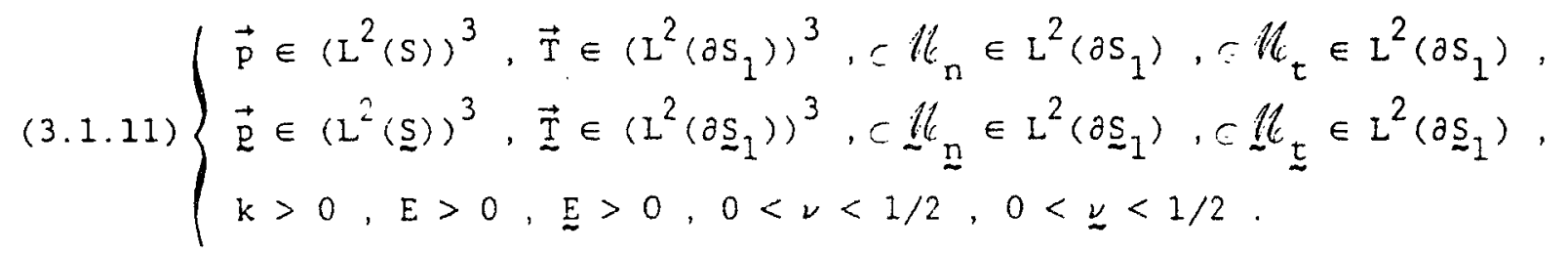

Then, the problem (3.1.7) has one and only one solution.

Proof: We give the main lines of this proof and we refer to [10] for more details. It takes three steps:

Step 1 : The space $W_{E \ell}$, defined by relation $(3.1 .6)$, is a closed subspace of the space

(3.1.12) $\quad E=\left[H^{1}(S)\right]^{2} \times H^{2}(S) \times\left[H^{1}(\underline{S})\right]^{2} \times H^{2}(S)$

equipped with the norm

$$
(3.1 .13)\left\{\begin{aligned}
\|(\vec{v} ; \vec{v})\|_{E}= & \left\|v_{1}\right\|_{H^{1}(S)}^{2}+\left\|v_{2}\right\|_{H^{1}(S)}^{2}+\left\|v_{3}\right\|_{H^{2}(S)}^{2} \\
& \left.+\left\|v_{\sim_{1}}\right\|_{H^{1}(\underline{S})}^{2}+\left\|v_{\sim_{2}}\right\|_{H^{1}(S)}^{2}+\left\|\underline{\sim}_{3^{\prime}}\right\|_{H^{2}(S)}^{2}\right)^{1 / 2} .
\end{aligned}\right.
$$

Step 2 : In the space $\mathrm{W}_{E \ell}$, the functional

$$
\begin{aligned}
& |(\vec{v} ; \vec{v})|_{W_{E \ell}}=\left\{\int_{S}\left[e_{\alpha \beta}(\vec{v}) e_{\alpha \beta}(\vec{v})+K_{\alpha \beta}(\vec{v}) K_{\alpha \beta}(\vec{v})\right] d S\right. \\
& +\int_{\underline{S}}[\underbrace{}_{\alpha \beta}(\vec{v}) e_{\alpha \beta}(\vec{v})+{\underset{\sim}{K}}_{\alpha \beta}(\vec{v}) \underset{\sim \alpha \beta}{K}(\vec{v})] d \underset{\sim}{S} \\
& +b[(\vec{v} ; \vec{v}),(\vec{v} ; \underline{v})]\}^{1 / 2}
\end{aligned}
$$

is an equivalent norm to the usual norm (3.1.13).

Step 3 : From Step 2, we deduce the $W_{E \ell}$-ellipticity of the bilinear form

$$
a[(. ; .),(. ; .)]+k b[(. ; .),(. ; .)]
$$

for any constant $k>0$. To conclude, it remains to apply LAX-MILGRAM lemma.

Remarque 3.1.1 : The assumption $k=$ constant $>0$ is not essential. One can assume that $k$ is a smooth function of the arc length $x$ along $\Gamma$ with $k(x)>0$. 


\section{2 - The case of a rigid hinge}

By taking into account the junction conditions $(2.3 .8)(2.3 .9)$ of the hinge, the space of the kinematically admissible fields is now :

$$
w_{R i g}-\left\{(\vec{w} ; \underline{w}) \in v \times \underline{v}, \vec{w}=\vec{w} \text { on } \Gamma, w_{3, n}+w_{3, \underline{n}}=0 \text { on } \Gamma\right\} \text {. }
$$

The variational formulation of the junction problem of two plates with a rigid hinge can be obtained by similarity with the previous one (see section 3.1 ); it is given by

(3.2.1) $\left\{\begin{array}{l}\text { Find }(\vec{u} ; \vec{u}) \in W_{R i g} \text { such that } \\ a[(\vec{u} ; \vec{u}),(\vec{v} ; \vec{v})]=\ell(\vec{v} ; \vec{v}), \forall(\vec{v} ; \vec{v}) \in W_{R i g}\end{array}\right.$

where $a[(. ;),(. ;)]$ and $\ell(. ;)$ are the bilinear and Iinear forms defined by relations $(3.1 .8)$ and $(3.1 .10)$. We prove:

Theorem 3.2.1 : Under the assumptions of Theorem 3.1.1, problem (3.2.1) has one and only one solution.

Proof : This proof is similar to that of Theorem 3.1.1. Briefly, we consider the following steps :

Step 1 : The space " ${ }_{\mathrm{Rig}}$ is a closed subspace of the space E defined by (3.1.12).

Step 2 : Upon the space $W_{R i g}$, the semi-norm

$(3.2 .2)\left\{\begin{aligned}|(\vec{v} ; \vec{v})|_{W_{R i g}} & =\left\{\int_{S}\left[e_{\alpha \beta}(\vec{v}) e_{\alpha \beta}(\vec{v})+K_{\alpha \beta}(\vec{v}) K_{\alpha \beta}(\vec{v})\right] d S\right. \\ & \left.+\int_{\underline{S}}[\underbrace{}_{\alpha \beta}(\vec{v}) e_{\alpha \beta}(\vec{v})+\underline{x}_{\alpha \beta}(\vec{v}) \underline{K}_{\alpha \beta}(\vec{v})] d \underline{S}\right]^{1 / 2}\end{aligned}\right.$

is equivalent to the norm (3.1.13).

Step 3 : Then, the bilinear form $a[(. ;),.(. ;)]$ is continuous, symmetric and $W_{R i g}$ -elliptic, and the linear form $l(.,$.$) is continuous on W_{R i g}$. We conclude by using the LAX-MILGRAM theorem.

\section{3 - Study of the behaviour of an elastic hinge when $k \rightarrow \infty$}

The next theorem justifies the assertion of Remark 2.2.1.

Theorem 3.3.1 : Let $\left(\vec{u}^{k}, \vec{u}^{k}\right)$ be the solution of problem $(3.1 .7)$ and let $(\vec{u} ; \underline{\vec{u}})$ be the solution of problem $(3.2 .1)$. Then $(\vec{u} ; \overrightarrow{\underline{u}})$ is strongly convergent to $(\vec{u}, \vec{u})$ in $w_{E l}$ when $\mathrm{k}$ goes to $+\infty$. 
Proof : It takes four steps.

Step 1 : Weak convergence of $(\overrightarrow{\mathrm{u}} ; \overrightarrow{\mathrm{u}} \mathrm{k})$ in $\mathrm{W}_{\mathrm{E} \ell}$ :

By using the continuity of the linear form $\ell(\ldots)$ on $W_{E \ell}$ and the $W_{E \ell}$ - ellipticity of the bilinear form

$$
a_{k}[(. ;),(. ;)]=a[(. ;),(. ;)]+k b[(. ;),(. ;)]
$$

we obtain

$$
\left\|\left(\vec{u}^{k} ; \vec{u}^{k}\right)\right\|_{W_{E \ell}} \leq \frac{\|\ell\|}{\alpha}
$$

as soon as $k \geq \alpha$ with $\alpha=\min \left(\frac{\mathrm{Ee}}{1-\nu^{2}} ; \frac{E e^{3}}{12\left(1-\nu^{2}\right)} ; \frac{E \mathrm{E}}{1-\nu^{2}} ; \frac{\mathrm{Ee}^{3}}{12\left(1-\nu^{2}\right)}\right)$.

Since the sequence $\left(\vec{u}^{k} ; \vec{u}^{k}\right)$ is bounded in $W_{E L^{*}}$, we can extract a subsequence, still noted $\left(\vec{u}^{k} ; \vec{u}^{k}\right)$ which is weakly convergent to $\left(\vec{u}^{*} ; \vec{u}^{*}\right)$ in $W_{E l}$.

Step $2:\left(\vec{u}^{*} ; \vec{u}^{*}\right) \in \cdot w_{R i g}$

By choosing $(\vec{v} ; \vec{v})=\left(\vec{u}^{k} ; \vec{u}^{k}\right)$ in $(3.1 .7)$, we get

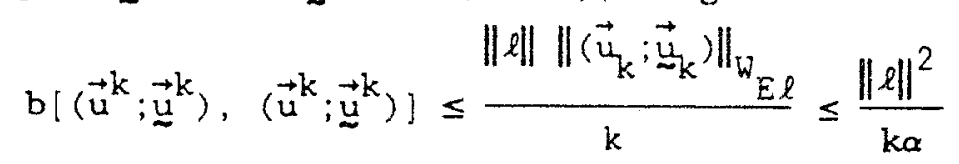

The application

$$
\left(\vec{u}^{k} ; \vec{u}^{k}\right) \rightarrow \underline{u}\left[\left(\vec{u}^{k} ; \vec{u}^{k}\right),\left(\vec{u}^{k} ; \vec{u}^{k}\right) j\right.
$$

is convex and continuous upon $\mathrm{H}^{2}(\mathrm{~S}) \times \mathrm{H}^{2}(\mathrm{~S})$ : then, it is lower weakly semi-continuous, so that for $k \rightarrow+\infty$, we obtain $b\left[\left(\vec{u}^{*} ; \vec{u}^{*}\right),\left(\vec{u}^{*} ; \vec{u}^{*}\right)\right]=0, i . e$.

$$
u_{3, n}^{*}=u_{3, n}^{*} \text { on } \Gamma \text { which is the expected result. }
$$

$\underline{\text { Step } 3}:\left(\vec{u}^{*} ; \vec{u}^{*}\right)=(\vec{u} ; \vec{u})$

Since $W_{R i g} \subset W_{E l}$, the equation (3.1.7) implies

$$
a\left[\left(\vec{u}^{k} ; \vec{u}^{k}\right),(\vec{v} ; \vec{v})\right]=\ell(\vec{v} ; \vec{v}), \forall(\vec{v} ; \vec{v}) \in W_{R i g}
$$

so that we find for $k \rightarrow+\infty$ :

$$
\left\{\begin{array}{l}
\left(u^{*}, \vec{u}^{*}\right) \in W_{R i g} \\
a\left[\left(\vec{u}^{*} ; \vec{u}^{*}\right),(\vec{v} ; \vec{v})\right]=\ell(\vec{v} ; \vec{v}), \forall(\vec{v} ; \vec{v}) \in W_{R i g}
\end{array}\right.
$$

Since this problem has $(\vec{u} ; \vec{u})$ as unique solution, we get the result.

Step 4 : Strong convergence of $\left(\vec{u}^{k}, \vec{u}_{k}\right)$ in $W_{E \ell}$

We have from the step 3 of the proof of theorem 3.1 .1 : 


$$
\begin{aligned}
0 & \leq d i(\vec{u} ; \vec{u})-\left(\vec{u}^{k} ; \vec{u}^{k}\right) \|_{W_{E}}^{2} \\
& \leq a_{k}\left[\left(\vec{u}^{k} ; \vec{u}^{k}\right),\left(\vec{u}^{k} ; \vec{u}^{k}\right)\right]-2 a_{k}\left[(\vec{u} ; \underline{u}),\left(\vec{u}^{k} ; \vec{u}^{k}\right)\right]+a_{k}[(\vec{u} ; \vec{u}),(\vec{u} ; \underline{\vec{u}})] .
\end{aligned}
$$

But, for $(\vec{u} ; \vec{u})$ solution of the rigid problem $(3.2 .1)$, we have

$$
a_{k}\left[(\vec{u} ; \vec{u}),\left(\vec{u}^{k} ; \vec{u}^{k}\right)\right]=a\left[(\vec{u} ; \vec{u}),\left(\vec{u}^{k} ; \vec{u}^{k}\right)\right]
$$

so that the previous inequalities become

$$
\begin{aligned}
0 & \leq d\left\|(\overrightarrow{\mathrm{u}} ; \underline{\vec{u}})-\left(\overrightarrow{\mathrm{u}}^{\mathrm{k}} ; \overrightarrow{\mathrm{u}}^{\mathrm{k}}\right)\right\|_{W_{E \ell}}^{2} \\
& \leq \ell\left(\overrightarrow{\mathrm{u}}^{\mathrm{k}} ; \overrightarrow{\mathrm{u}}^{\mathrm{k}}\right)-2 \mathrm{a}\left[(\overrightarrow{\mathrm{u}} ; \underline{\vec{u}}),\left(\vec{u}^{\mathrm{k}} ; \vec{u}^{\mathrm{k}}\right)\right]+\ell(\overrightarrow{\mathrm{u}} ; \overrightarrow{\mathrm{u}}) .
\end{aligned}
$$

It remains to use the weak convergence of $\left(\vec{u}^{k} ; \vec{u}^{k}\right)$ to $(\vec{u} ; \vec{u})$ to prove the convergence to zero of the right hand member; this result implies the strong convergence of $\left(\vec{u}^{k} ; \vec{u}^{k}\right)$ to $(\vec{u} ; \vec{u})$. Since this limite is independent of the subsequence into consideration, the result is valid for all the sequence $\left(\vec{u}^{k} ; \vec{u}^{k}\right)$.

\section{4 - APPROXIMATION BY CONFORMING FINITE ELEMENT METHODS}

This paragraph is devoted to the approximation of problems (3.1.7) and (3.2.1) by . conforming finite element methods. We consider two cases corresponding to an elastic hinge and a rigid hinge.

\section{1 - Approximation in case of an elastic hinge}

The approximation of the solution of problem (3.1.7) is realized by means of a conforming finite element method. Thus, we define a finite dimensional subspace

(4.1.1) $\quad w_{E \ell}^{h} \subset W_{E \ell}$

as follows

(4.1.2) $\quad w_{E \ell}^{h}=\left\{\left(\vec{w}_{h} ; \vec{w}_{h}\right) \in\left(\vec{v}_{h} ; \overrightarrow{\vec{v}}_{h}\right), \vec{w}_{h}(M)-\vec{w}_{h}(M) \quad \forall M \in \Gamma\right\}$

with

$$
\left\{\begin{array}{l}
v_{h}=v_{h 1} \times v_{h 1} \times v_{h 2} ; \vec{v}_{h}=v_{h 1} \times v_{h 1} \times v_{h 2} \\
v_{h 1} \subset v_{1}, v_{h 2} \subset v_{2} ; v_{h 1} \subset H^{1}(\underline{s}) ; v_{h 2} \subset \mathrm{H}^{2}(\underline{s}) .
\end{array}\right.
$$

The spaces $V_{h l}$ and $V_{h l}$ are constructed from HERMITE triangles of type ( $3^{\prime}$ ) (see figure 4.1.1) while the spaces $v_{h 2}$ and $v_{h 2}$ are constructed from the $\left(f^{1}\right.$-class reduced HSIEH-CLOUGH-TOCHER triangles (see figure 4.1.2).

The inclusion (4.1.1) implies that the following discrete problem associated to the continuous problem (3.1.7) has one and only one solution : 
(4.1.3) $\left\{\begin{array}{l}\text { Find }\left(\vec{u}_{h}^{*} ; \vec{u}_{h}^{*}\right) \in w_{E \ell}^{h} \text { such that } \\ a\left[\left(\vec{u}_{h}^{*} ; \vec{u}_{h}^{*}\right),\left(\vec{v}_{h} ; \vec{v}_{h}\right)\right]+k b\left[\left(\vec{u}_{h}^{*} ; \vec{v}_{h}^{*}\right),\left(\vec{v}_{h} ; \vec{v}_{h}\right)\right]=\ell\left(\vec{v}_{h} ; \vec{v}_{h}\right), \forall\left(\vec{v}_{h}, \vec{v}_{h}\right) \in w_{E l}^{h}\end{array}\right.$

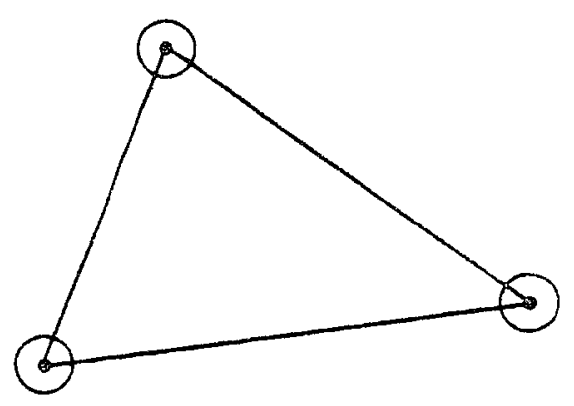

Figure 4.1.1: Hermite Triangle of type (3')

(see $[11$, page 681)

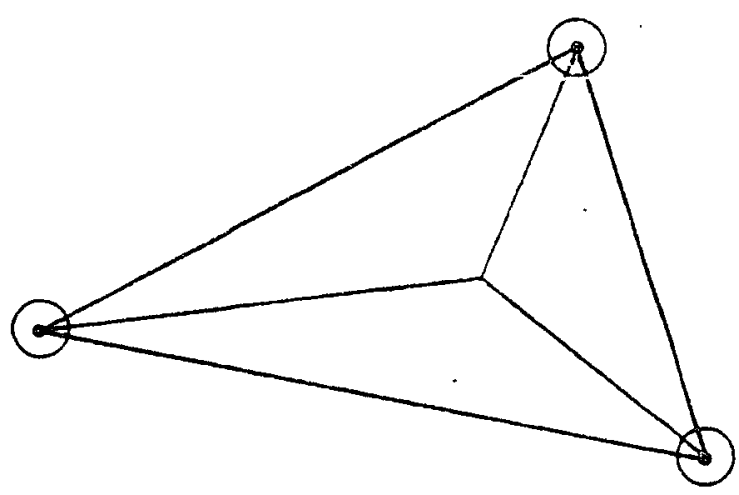

Figure 4.1.2: Reduced H.C.T. -Triangle

(see [11, page 357])

But the exact computation of the integrals which appear in relation (4.1.3) is generally impossible. This makes mandatory. the use of numerical integration techniques. This leads to the definition of a second discrete problem :

(4.1.4) $\left\{\begin{array}{l}\text { Find }\left(\vec{u}_{h} ; \vec{u}_{h}\right) \in w_{E l}^{h} \text { such that } \\ \text { h }\end{array}\right.$

$a_{h}\left[\left(\vec{u}_{h} ; \vec{w}_{h}\right),\left(\vec{v}_{h} ; \vec{v}_{h}\right)\right]+k b\left[\left(\vec{u}_{h} ; \vec{w}_{h}\right),\left(\vec{v}_{h} ; \vec{v}_{h}\right)\right]-\ell_{h}\left(\vec{v}_{h} ; \vec{v}_{h}\right), \forall\left(\vec{v}_{h}, \vec{v}_{h}\right) \in h_{E l}^{h}$

where the forms $a_{h}(\ldots)$ and $\ell_{h}($.$) are deduced from forms a(.$,$) and l($.$) through$ the use of appropriate numerical integration schemes. Moreover we will see in section 4.3 .2 that the bilinear from $b(.,$.$) can be computed exactly.$ 
It remains to prove the existence, uniqueness and convergence of the solution $\left(\vec{u}_{h} ; \vec{u}_{h}\right)$ to the solution $(\vec{u} ; \vec{u})$ of the continuous problem (3.1.5). Such a study follows the lines of [12] and is detailed in [10].

For instance, when the boundaries $\partial \mathrm{S}_{1}$ and $\partial \underline{\mathrm{S}}_{1}$ are unloaded and when the data and the solution $(\vec{u} ; \vec{u})$ of the continuous problem (3.1.7) are sufficiently smooth, then such methods allow to prove that there exist a constant $C$, independent of $h$, such that :

(4.1.5) $\quad\left\|(\vec{u} ; \vec{u})-\left(\vec{u}_{h} ; \vec{u}_{h}\right)\right\|_{E} \leq C h$

as soon as the numerical integration schemes used to compute the integrals upon $S$ and $\underset{S}{S}$ are exact for polynomials

i) of degree 4 for the membrane terms (there exist such schemes involving 6 nodes per triangle)

ii) of degree 2 for the bending terms upon each subtriangle of the HCT triangles (there exist such schemes involving 3 nodes).

When the boundaries $\partial S_{1}$ and $\partial{\underset{\sim}{1}}_{1}$ are loaded, we have to introduce some appropriate numerical integration technique for approximating the integrals along these boundaries. This study can be done as in [13] so that the estimate is still valid.

\section{2 - Approximation in case of a rigid hinge}

The formulations are similar to those of section 4.1, except for the definition of the finite dimensional subspace $W_{R i g}^{h}$ which has to satisfy :

(4.2.1) $W_{R i g}^{\text {h }} \subset w_{R i g}$.

It is defined by

(4.2.2) $\left\{\begin{aligned} w_{R i g}^{h}=\left(\left(\vec{w}_{h} ; \vec{w}_{h}\right) \in\left(\vec{v}_{h} ; \vec{v}_{h}\right),\right. & \vec{w}_{h}(M)-\vec{w}_{h}(M) \\ & \left.\text { and } w_{h 3, n}(M)+w_{h 3, \underline{n}}(M)=0, \forall M \in \Gamma\right\}\end{aligned}\right.$

so that the second discrete problem associated to the use of numerical integration scheme can be stated as follows (compare with (4.1.4)) :

(4.2.3) $\left\{\begin{array}{l}\text { Find }\left(\vec{u}_{h} ; \vec{w}_{h}\right) \in w_{R i g}^{h} \text { such that } \\ a_{h}\left[\left(\vec{u}_{h} ; \vec{u}_{h}\right),\left(\vec{v}_{h} ; \vec{v}_{h}\right)\right]=\ell_{h}\left(\vec{v}_{h} ; \vec{v}_{h}\right), \forall\left(\vec{v}_{h}, \vec{v}_{h}\right) \in w_{R i g}^{h} .\end{array}\right.$

As in section 4.1, the methods developped in. [10] [12] and [13] would allow us to obtain an a priori estimate of type (4.1.5) as soon as the data, the solution and the numerical integration schemes are sufficiently regular. 
Remark 4.2.1: Of course, the solutions $\left(\vec{u}_{n} ; \vec{u}_{h}\right)$ of problems $(4.1 .4)$ and (4.2.3) are different ; in particular, the solution of problem (4.1.4) is depending of $k$.

\section{3 - Implementation in case of an elastic hinge}

Upon each plate $S$ and $S$ the implementation follows the usual lines, i.e., computation of local matrices, element by element, and assemblage into the global matrices as the computations proceed. In fact the only new part of such an implementation is related to the hinge. There are two new things to consider:

\subsection{1 : Taking the condition $\vec{w}_{h}(M)=\vec{w}_{h}(M), \forall M \in \Gamma$ into account}

This junction condition is an essential part of the definition of the space (4.1.2). By choosing the reference systems $\left(\vec{n}, \vec{t}, \vec{e}_{3}\right)$ and $\left(\vec{n}, \overrightarrow{\underline{t}}, \vec{e}_{3}\right)$ along $\Gamma$, respectively attached to the plates $S$ and $\underline{S}$, the condition $\vec{w}_{h}(M)=\vec{w}_{h}(M), \forall M \in \Gamma$,
implies (see $(2.2 .7)$ ).:

$(4.3 .1)\left\{\begin{array}{l}\underline{w}_{h \underline{n}}=w_{h n} \cos \theta-w_{h 3} \sin \theta \\ {\underset{w h}{h t}}_{\sim}=-w_{h t} \\ {\underset{w}{h 3}}_{h 3}=-w_{h n} \sin \theta-w_{h 3} \cos \theta .\end{array}\right.$

Moreover, the condition $\vec{w}_{h}(M)=\vec{w}_{h}(M), \forall M \in \Gamma$, implies $\vec{w}_{h, t}(M)=-\vec{w}_{h, t}(M), \forall M \in \Gamma$.
Thus, it follows from $(4.3 .1):$

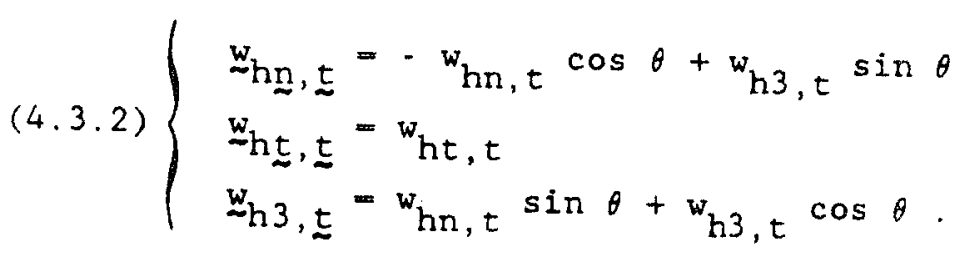

The restrictions of these functions $w_{h n}, w_{h t}, w_{h 3}, w_{h n}, w_{h t}$ and $w_{h 3}$ to the hinge $\Gamma$ are polynomials of order 3 in one variable upon each side $a_{1} a_{2}$ located on $\Gamma$. Then, to satisfy relations $(4.3 .1)$ and $(4.3 .2)$ at any point of $\Gamma$, it suffices to satisfy them at any vertex located on $\Gamma$. Thus the main unknowns of the problem can be chosen as the values of

(4.3.3) $\left\{\begin{array}{l}u_{h n} ; u_{h t} ; u_{h 3} ; u_{h n, n} ; u_{h n, t} ; u_{h t, n} ; u_{h t, t} ; u_{h 3, n} ; u_{h 3, t} \\ u_{h n, n} ; u_{h t, n} ; u_{h 3, n} .\end{array}\right.$

at any vertex of the mesh located on $\Gamma$. Of course the relations (4.3.1) and (4.3.2) are symmetrical in $w$ and $w$ so that we could permute the roles of $u$ and $\underline{\underline{u}}$ in the
relation ( 4.3 .3$)$. 
4.3 .2 : Exact computation of $b\left[\left(\vec{u}_{h} ; \vec{w}_{h}\right),\left(\vec{v}_{h} ; \vec{w}_{h}\right)\right]$

According to relation (3.1.9), we have

$$
\mathrm{b}\left[\left(\overrightarrow{\mathrm{u}}_{\mathrm{h}} ; \overrightarrow{\mathrm{u}}_{\mathrm{h}}\right) ;\left(\vec{v}_{\mathrm{h}} ; \overrightarrow{\mathrm{w}}_{\mathrm{h}}\right)\right]=\int_{\mathrm{T}}\left(\mathrm{u}_{\mathrm{h} 3, \mathrm{n}}+\mathrm{u}_{\mathrm{h} 3, \mathrm{n}}\right)\left(\mathrm{v}_{\mathrm{h} 3, \mathrm{n}}+\mathrm{v}_{\mathrm{h} 3, \mathrm{n}}\right) \mathrm{d} \gamma .
$$

By definition of the reduced H.C.T.-triangle we obtain

(4.3.5) $\quad u_{h 3, n}(M)=\lambda u_{h 3, n}\left(a_{1}\right)+(1-\lambda) u_{h 3, n}\left(a_{2}\right)$

for any point $M$ belonging to a side $a_{1} a_{2}$ of a triangle, this side being located upon the hinge $\Gamma$.

Of course, we have similar relations for ${\underset{\sim}{h} 3, n}_{n}, v_{h 3, n}$ and ${\underset{\sim}{h} 3, \underline{n}}_{\mathfrak{n}}$. Thus, it is easy to check that

$(4.3 .6)$

$$
\int^{b[(\vec{u}}
$$

$\left.\left(\vec{u}_{h} ; \vec{w}_{h}\right) ;\left(\vec{v}_{h} ; \vec{v}_{h}\right)\right]$

$$
\begin{aligned}
=\sum_{\left[a_{1}, a_{2}\right] \in \Gamma} \quad \frac{\frac{1}{3}\left[u_{h 3, n}\left(a_{1}\right)-u_{h 3, n}\left(a_{1}\right)\right]\left[v_{h 3, n}\left(a_{1}\right)-v_{h 3, n}\left(a_{1}\right)\right]}{+\frac{1}{6}\left[u_{h 3, n}\left(a_{1}\right)-u_{h 3, n}\left(a_{1}\right)\right]\left[v_{h 3, n}\left(a_{2}\right)-v_{h 3, n}\left(a_{2}\right)\right]}
\end{aligned}
$$$$
+\frac{1}{6}\left[u_{h 3, n}\left(a_{2}\right)-u_{h 3, n}\left(a_{2}\right)\right]\left[v_{h 3, n}\left(a_{1}\right)-y_{h 3, n}\left(a_{1}\right)\right]
$$$$
\left.+\frac{1}{3}\left[u_{h 3, n}\left(a_{2}\right)-u_{n, n}\left(a_{2}\right)\right]\left[v_{h 3, n}\left(a_{2}\right)-w_{h 3, n}\left(a_{2}\right)\right]\right\}
$$

These contributions are easy to assemble in the global rigidity matrix.

\section{4 - Implementation in case of a rigid hinge}

The main differences with the previous case are

i) the additional condition $w_{h 3, n}(M)+w_{h 3, n}(M)=0, \forall M \in \Gamma$ which appears in the definition (4.2.2) of the space $w_{\mathrm{Rig}}^{\mathrm{h}}$;

ii) the absence of term $b[. ;$.$] in the statement of problem (4.2.3).$

The comparison with section 4.3 .1 shows immediately that conditions (4.3.1) and (4.3.2) are still valid and that they have to be complemented by the additional

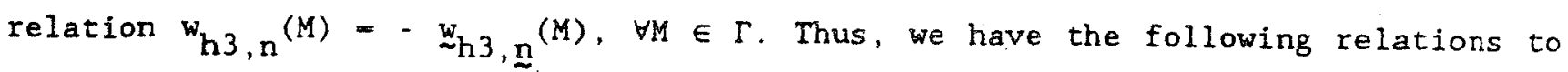
satisfy at any point $M \in \Gamma$ :

$(4.4 .1)\left\{\begin{array}{l}\underline{w}_{h \underline{ }}=w_{h n} \cos \theta-w_{h 3} \sin \theta ; w_{h t}-w_{h t} ; w_{h 3}-w_{h n} \sin \theta-w_{h 3} \cos \theta \\ \underline{w}_{h n, t}=-w_{h n, t} \cos \theta+w_{h 3, t} \sin \theta ; w_{h t, t}=w_{h t, t} ; \\ \underline{w}_{h 3, \underline{n}}=-w_{h 3, n} .\end{array}\right.$ 
Thus similar arguments to those used in section 4.3 prove that we can choose lit following set of in unknows:

(4.4.2) $\left\{\begin{array}{l}u_{h n} ; u_{h t} ; u_{h 3} ; u_{h n, n} ; u_{n n, t} ; u_{n t, n} ; u_{h t, t} ; u_{h 3, n} ; u_{n 3, t} \\ u_{h n, n} ; u_{n t, n}\end{array}\right.$

at any vertex of the located on $\Gamma$. In particular, we have used that the restrictions of $w_{h 3, n}$ and $w_{h 3, n}$ to $\Gamma$ are locally polynomials of degree one.

\section{5 - NUMERICAL EXAPIPLES}

We have considered the three following examples which have been implemented in MODULEF (see [14]).

\section{1 - Example of a folded cantilever plate with rigid hinge, subjected to a line load at its tip}

This example is extracted from [2] and [15, test $n^{\circ} \mathrm{A}-24$ ].

According to figure 5.1.1, we consider a rigid junction of two plates : the first one is clamped along the side aa' while the second one is loaded along the side cc' by a line density of forces

$$
P_{Z}=-1 \cdot 2 b / \text { in }
$$

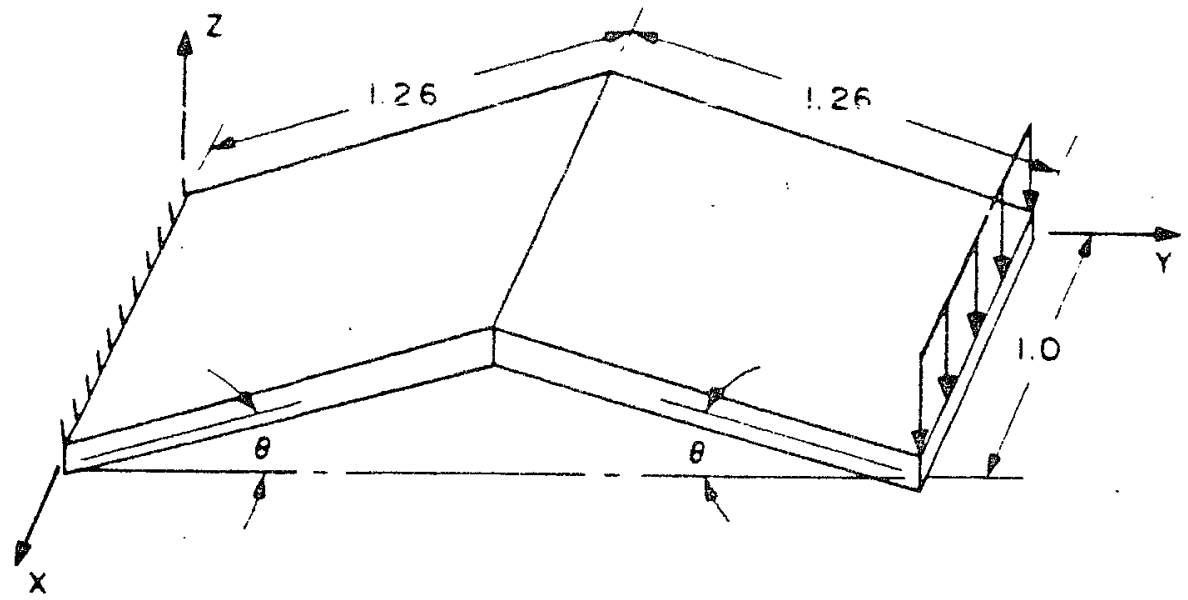

$$
\begin{array}{ll}
e=0.124 \text { in } & E=3 \times 10^{7} \mathrm{psi} \\
\theta=30^{\circ} & \nu=0
\end{array}
$$

\section{Figure 5.1.1: The folded cantilever plate}

For this study we have used the mesh shown on the figure 5.1.2. Upon this figure, we represent the deformed configuration of the cantilever plate which is in good agreement with the results reported in [15].

In particular, we find $Z_{A}=-0.000837274$ in. and $Z_{B}-0.000837166$ in. 


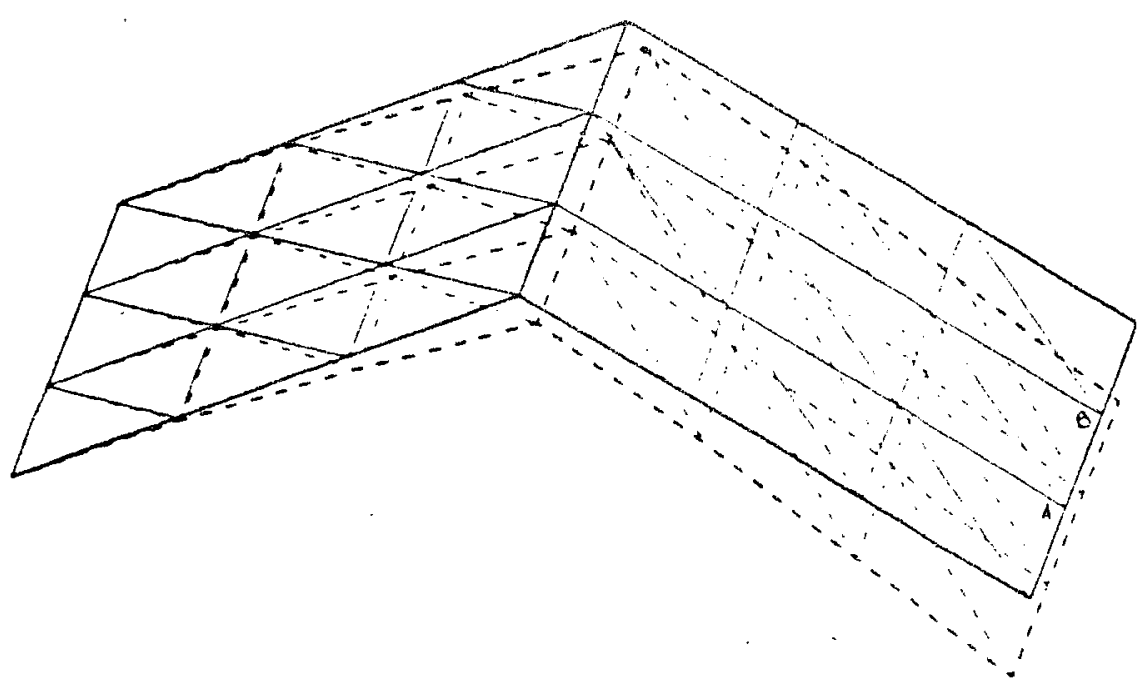

Figure 5.1.2 : The cantilever before loading (-)

and after loading (-- ) for a rigid hinge

\section{2 - Example of a multifolded plate with rigid hinges}

This example was previously considered by (16) (17). With notations of figure 5.2.1, the structure is clamped along the side a'f' and loaded along the hinges b: two types of line densities

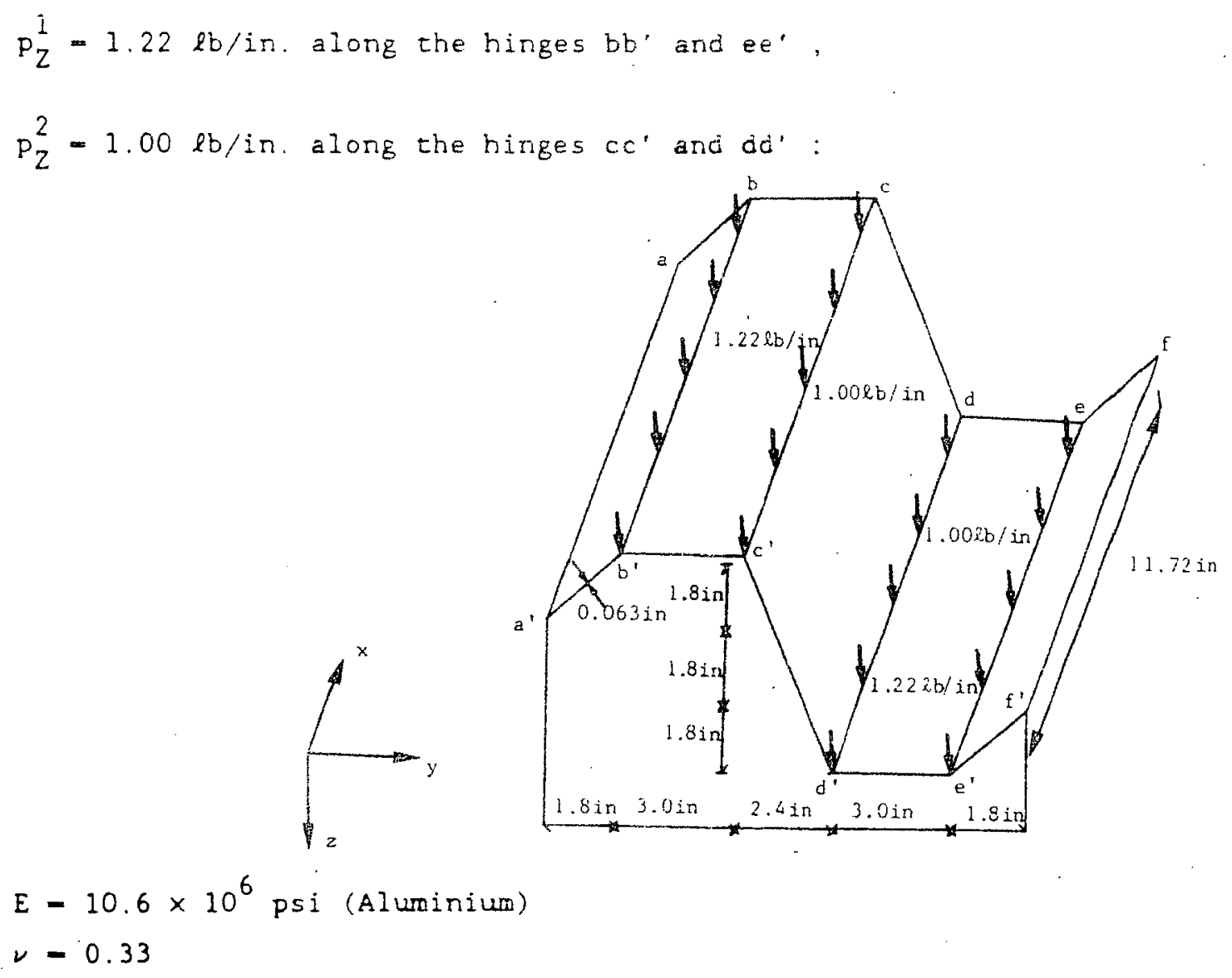

Figure 5.2.1: Hultifolded plate 
For this study we have used the wesh shown on the figure 5.2 .2 .

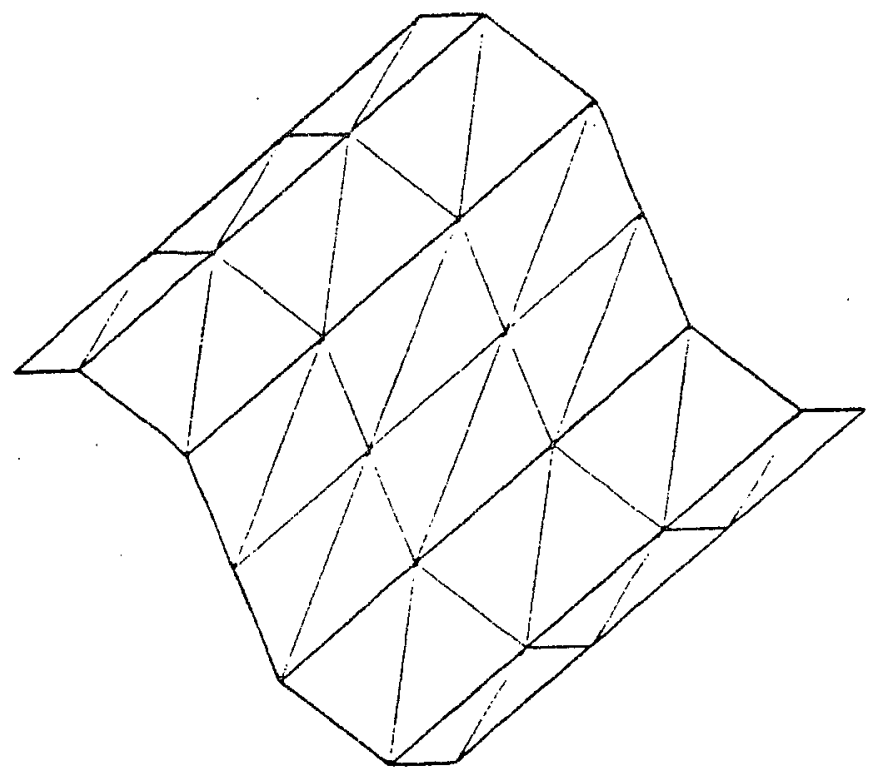

Figure 5.2.2.: Mesh in use

The experimental results and the analytical solution are extracted from (16); they are included with our numerical results in the tableau 5.2.1.

\begin{tabular}{|c|c|c|c|c|c|c|}
\hline \multicolumn{7}{|c|}{${ }^{\mid \prime z}$ (i.i.) } \\
\hline Points & $a$ & $b$ & $c$ & $d$ & e & $f$ \\
\hline Experimental & - & 0.0058 & 0.0013 & 0.0012 & 0.0063 & - \\
\hline Analytical & 0.0136 & 0.0080 & 0.0008 & 0.0008 & 0.0080 & 0.0136 \\
\hline Numerical & 0.0134 & 0.0070 & 0.0008 & 0.0008 & 0.0060 & 0.0122 \\
\hline
\end{tabular}

Tableau 5.2.1: Vertical displacement of points a to $f$

\section{3 - Example of a folded cantilever plate with elastic hinge subjected to a line load at its tip}

The data are identical to those of the first example except that now the hinge is supposed to be elastic. The figure 5.3.1 presents the deformed configuration when $k=10^{4} \mathrm{lb}$. and $k=10^{3} \mathrm{lb}$. 


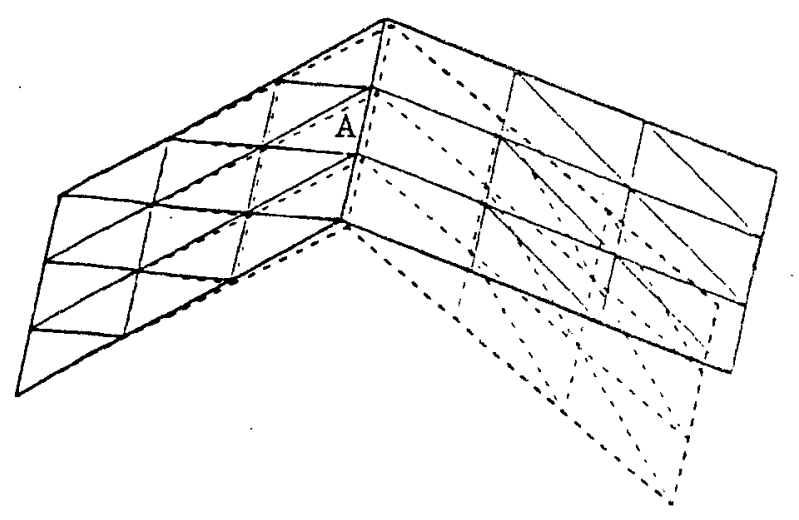

$k=10^{4} 2 b$.

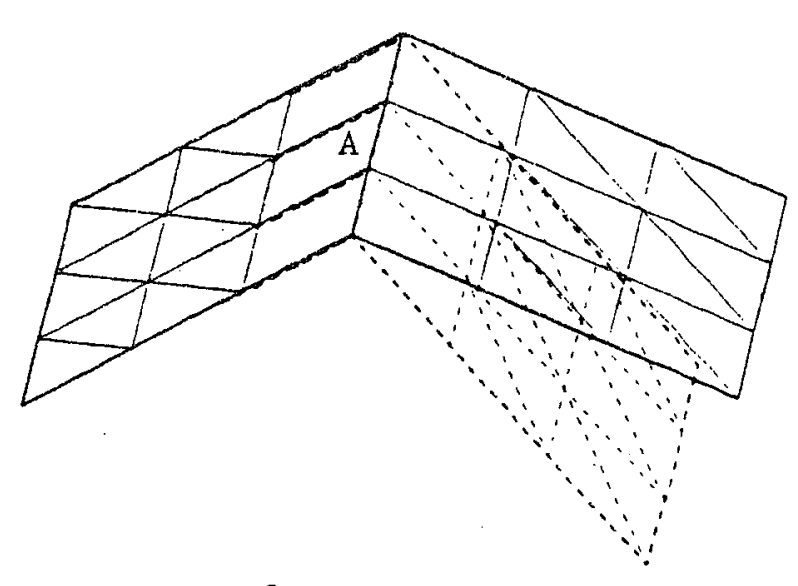

$k=10^{3} \ell b$.

Figure 5.3.1: The folded cantilever before loading $(-)$ and after loading $(-\rightarrow)$ for an elastic hinge

Finally, figure 5.3 .2 shows the jump of the rotation $(\vec{\Omega} \cdot \vec{\Omega}) \cdot \vec{t}=\left(u_{3, n}+\underline{u}_{3, \underline{n}}\right)$ at point $A \in \Gamma$ (see figure 5.3.1). We can check that the hinge behaves:

i) as a knee-joint when the coefficient $k$ is small;

ii) as a rigid hinge when the coefficient $k$ is very large.

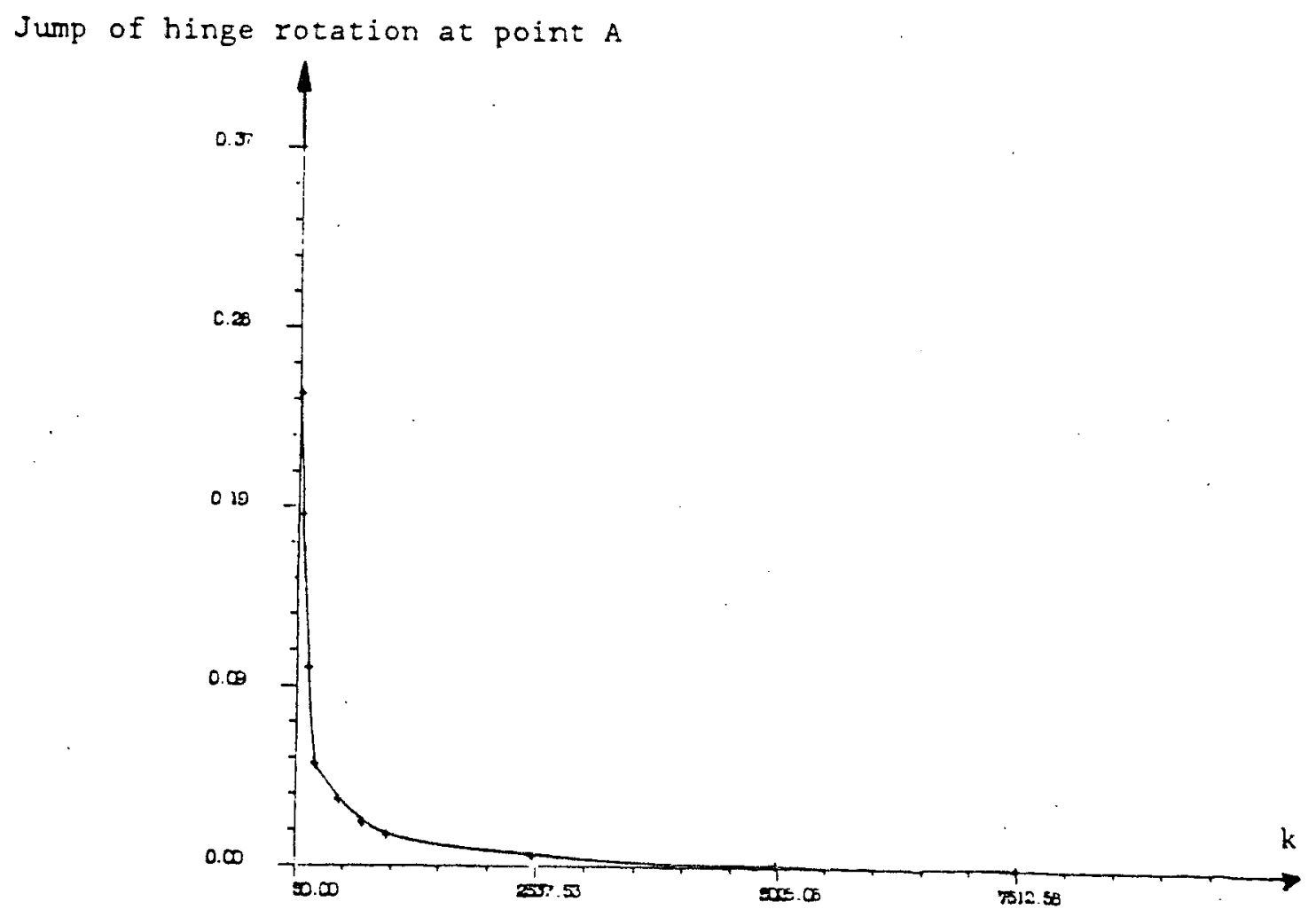

Figure 5.3.2: Jump of the hinge rotation at point A

as a function of coefficient $k$ 
This study gives a precise variational formulation of the junction problem of two plates by using appropriate functional spaces. From this formulation, we have approximated the solution by conforming finite element methods which are easy to implement.

By using the same ideas we are extending these results to the junction of two shells having general shapes.

\section{REFERENCES}

[1] E.H. BAKER, L. KOVALEVSKY, F.L. RISH : Structural Analysis of Shelis (R.E. KRIEGER Publishing Company, Huntington, New York, 1981).

[2] K.J. BATHE, L.W. HO : Some results in the analysis of thin shell structures, in : W. Wunderlich et al., ed., Nonlinear Finite Element Analysis in Structural Mechanics (Springer-Verlag, Berlin, 1981), 122-150.

[3] K.J. BATHE : Finite Element Procedures in Engineering Analysis (Prentice Hall, Inc., Englewood Cliffs, New Jersey, 1982).

14] P.G. CIARLET : Modelling and numerical analysis of junctions between elastic structures in : Proceedings of the First International Conference on Industrial and Applied Mathematics, Paris (1987).

[5] P.G. CIARLET, H. Le DRET, R. NZENGWA : Modélisation de la jonction entre un corps élastique tridimensionnel et une plaque, C.R. Acad. Sci. Paris 305, Série I, (1987), 55-58.

[6] M. AUFRANC : Etudes numériques de raccords de structures élastiques de dimensions différentes, Rapports de Recherche INRIA $n^{\circ} 781$ (Février 1988).

[7] H. Le DRET : Modelling of a folded plate, Publications du Laboratoire d'Analyse Numérique de l'Université Pierre et Marie Curie, Nº R87017 (1987).

[8] H. Le DRET : Folded plates revisited, Publications du Laboratoire d'Analyse Numérique de I'Université Pierre et Marie Curie, NR87032 (1987).

[9] P. GERMaIN : Cours de Mécanique (Ecole Polytechnique, 1979).

[10] S. FAYOLLE : Sur l'analyse numérique de raccords de poutres et de plaques, Thèse de 3ème cycle, Université Pierre et Marie Curie - Paris VI (1987).

[11] P.G. CIARLET : The Finite Element Method for Elliptic Problems (North-Holland, Amsterdam, 1978). 
[12] M. BERNADOU : Convergence of conforming finite element methods for general she11 problems, Int. J. Engng. Sci. 18, (1980), 249-276.

11:: MeRNADOU : Méthodes numériques in: $R$. DAUTRAY and J.L. LIONS, Ed., Analyse Mathématique et Calcul Numérique pour les Sciences et les Techniques Tome 2, (Masson, Paris, 1985), 703-910.

[14] M. BERNADOU, P.L. GEORGE, A. HASSIM, P. JOLY, P. LAUG, A. PERRONNET, E. SAltel, D. STEER, G. VANDERBORCK, M. VIDRASCU, MODULEF : A Modular Finite Element Library (INRIA, Rocquencourt, 1986).

[15] ADINA : System verification manual (Report AE 83-5, 1983).

[16] A.C. SCORDELIS, E.I. CROY, I.R. STUBBS : Experimental and analytical study of a folded plate, J. Struct. Div. A.S.C.E., 87, ST8 (1961), 139-160.

[17] J.L. MEEK, H.S. TAN : A faceted shell element with Loof nodes, Int. J. Numer. Methods Eng. 23, (1986), 49-67. 
